\title{
Note sur les oscillations non radiales du modèle standard
}

\author{
H. Robe, P. Ledoux
}

\begin{abstract}
Abstract. - The non radial oscillations of the standard model are studied numerically for values of the degree $l$, of the spherical harmonics ranging from $I=2$ to $I=50$ in the case of the g modes and $I=2$ to 200 in the case of the $f$ and $p$ modes. It is found that for values $I>50$, the eigenvalues $\sigma^{2}$ of the $f$ and $p$ modes are nearly proportional to $I$ and that the amplitudes are only important in a superficial region, the narrower the higher the value of $I$. Moreover, for / in the range 400-800, the periods of the first $p$ modes in the case of the sun, supposed built on this model, vary from 3 to 5 minutes. The eigenvalues of the $g$ modes seem to be bounded and while the corresponding amplitude remains important in the whole model, it tends to increase considerably, especially for the $\mathrm{g}_{1}, \mathrm{~g}_{2}$ and $\mathrm{g}_{3}$ modes, in the central regions as / increases. This behaviour of the eigenfunctions could have interesting consequences for vibrational instability.
\end{abstract}

\section{Citer ce document / Cite this document :}

Robe H., Ledoux P. Note sur les oscillations non radiales du modèle standard. In: Bulletin de la Classe des sciences, tome 61, 1975. pp. 198-209;

doi : https://doi.org/10.3406/barb.1975.57908

https://www.persee.fr/doc/barb_0001-4141_1975_num_61_1_57908

Fichier pdf généré le 05/06/2020 


\title{
ASTROPHYSIQUE
}

\author{
Note sur les oscillations non radiales du modèle standard \\ H. ROBE et P. LEDOUX \\ Institut d'Astrophysique - Université de Liège
}

\begin{abstract}
The non radial oscillations of the standard model are studied numerically for values of the degree $l$, of the spherical harmonics ranging from $l=2$ to $l=50$ in the case of the $g$ modes and $l=2$ to 200 in the case of the $f$ and $p$ modes. It is found that for values $l>50$, the eigenvalues $\sigma^{2}$ of the $f$ and $p$ modes are nearly proportional to $l$ and that the amplitudes are only important in a superficial region, the narrower the higher the value of $l$. Moreover, for $l$ in the range $400-800$, the periods of the first $p$ modes in the case of the sun, supposed built on this model, vary from 3 to 5 minutes. The eigenvalues of the $g$ modes seem to be bounded and while the corresponding amplitude remains important in the whole model, it tends to increase considerably, especially for the $g_{1}, g_{2}$ and $g_{3}$ modes, in the central regions as $l$ increases. This behaviour of the eigenfunctions could have interesting consequences for vibrational instability.
\end{abstract}

\section{INTRODUCTION}

Bien que les oscillations non radiales des sphères gazeuses fassent l'objet d'une littérature déjà importante (pour une revue récente, voir Ledoux 1974), il semble que, mis à part les travaux de P. Smeyers (1970) et de Souffrin et al. (1972) traitant des modes $g^{-}$associés à une zone convective et deux notes de Wolff (1972 a, b) concernant quelques modes $p$ du soleil, une étude systématique des modes $p, f$ et $g^{+}$correspondant à des harmoniques sphériques de degré $l$ élevé fasse encore défaut.

Pourtant, l'étude de tels modes, et notamment des modes $p$, comme le souligne Wolff, peut être intéressante en vue d'expliquer les mouve- 
ments oscillatoires de période voisine de 5 minutes observés par Leighton et al. à la surface du soleil (pour une revue récente, voir Stein and Leibacher 1974). Il est vrai que pour $l$ élevé, les premiers de ces modes $p$ en tout cas, n'ont des amplitudes appréciables que dans les couches très extérieures qui peuvent dès lors être traitées comme planes, approximation souvent employée dans ce problème (pour une revue récente voir Michalitsanos 1973). Cette même approximation a aussi été utilisée fréquemment dans ce contexte pour les modes $g$ (MacKenzie 1971, Thomas Clark and Clark 1971, Worral 1972). Si cette approximation est justifiée lorsqu'il s'agit des modes $g$ trappés pour des raisons physiques dans une couche extérieure, par exemple au-dessus de la zone convective du soleil, elle le serait beaucoup moins pour les modes $g^{+}$associés à l'intérieur radiatif du soleil sous l'enve-

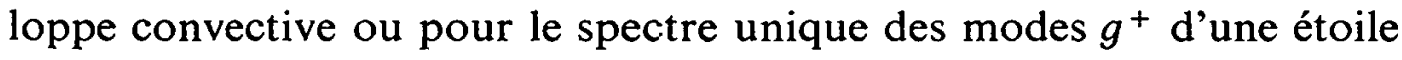
complètement radiative. En effet, négligeant comme Cowling (1971) la perturbation du potentiel gravifique, les équations pour les oscillations non radiales d'une étoile peuvent s'écrire avec les notations habituelles (P. Ledoux and Th. Walraven 1958) et après séparation du temps et des variables angulaires $\theta$ et $\varphi$ par le facteur $e^{i \sigma t} \cdot \mathrm{Y}_{l}^{m}(\theta, \varphi)$ :

$$
\begin{gathered}
\frac{d v}{d r}=\left[\frac{l(l+1)}{\sigma^{2}}-\frac{\rho r^{2}}{\gamma p}\right] \frac{p^{2 / \gamma}}{\rho} w \equiv \mathrm{K} w \\
\frac{d w}{d r}=\frac{1}{r^{2}}\left[\sigma^{2}+\mathrm{A} g\right] \frac{\rho}{p^{2 / \gamma}} v \equiv \mathrm{G} v
\end{gathered}
$$

où

$$
g=-\frac{1 d p}{\rho d r} ; \mathrm{A}=\frac{1 d \rho}{\rho d r}-\frac{1 d p}{\gamma p d r}
$$

$v$ et $w$ étant liés au déplacement radial $\delta r$ et à la perturbation eulérienne $p^{\prime}$ de la pression par

$$
v=p^{1 / \nu} r^{2} \delta r ; w=p^{\prime} / p^{1 / \gamma} .
$$

Or, Scuflaire (1974) a montré que dans le plan [log $\left.\sigma^{2}, r\right]$, excluant le cas de très grande concentration massique, les nœuds des modes $p$ se trouvent dans la région $\mathrm{G}>0, \mathrm{~K}<0$, et ceux des modes $g$ dans la région $\mathrm{G}<0, \mathrm{~K}>0$ définies par les courbes $\mathrm{G}=0, \mathrm{~K}=0$. La figure 1 représente celles-ci pour $l=2, l=25$ et $l=80$ dans le cas du polytrope $n=3$ pour $\gamma=5 / 3$, donc en équilibre radiatif stable partout. 
Lorsque $l$ augmente, la courbe $\mathrm{K}=0$ se déplace vers la droite c'est-à-dire vers $x=1$ et ceci implique que les nœuds des premiers modes $p$ se déplacent aussi vers la surface. Par contre, les nœuds des premiers modes $g$ peuvent a priori se trouver aux environs de la surface, ou dans une zone plus centrale ou distribués dans ces deux régions. Dans les derniers cas, l'approximation plane ne peut plus être valable.

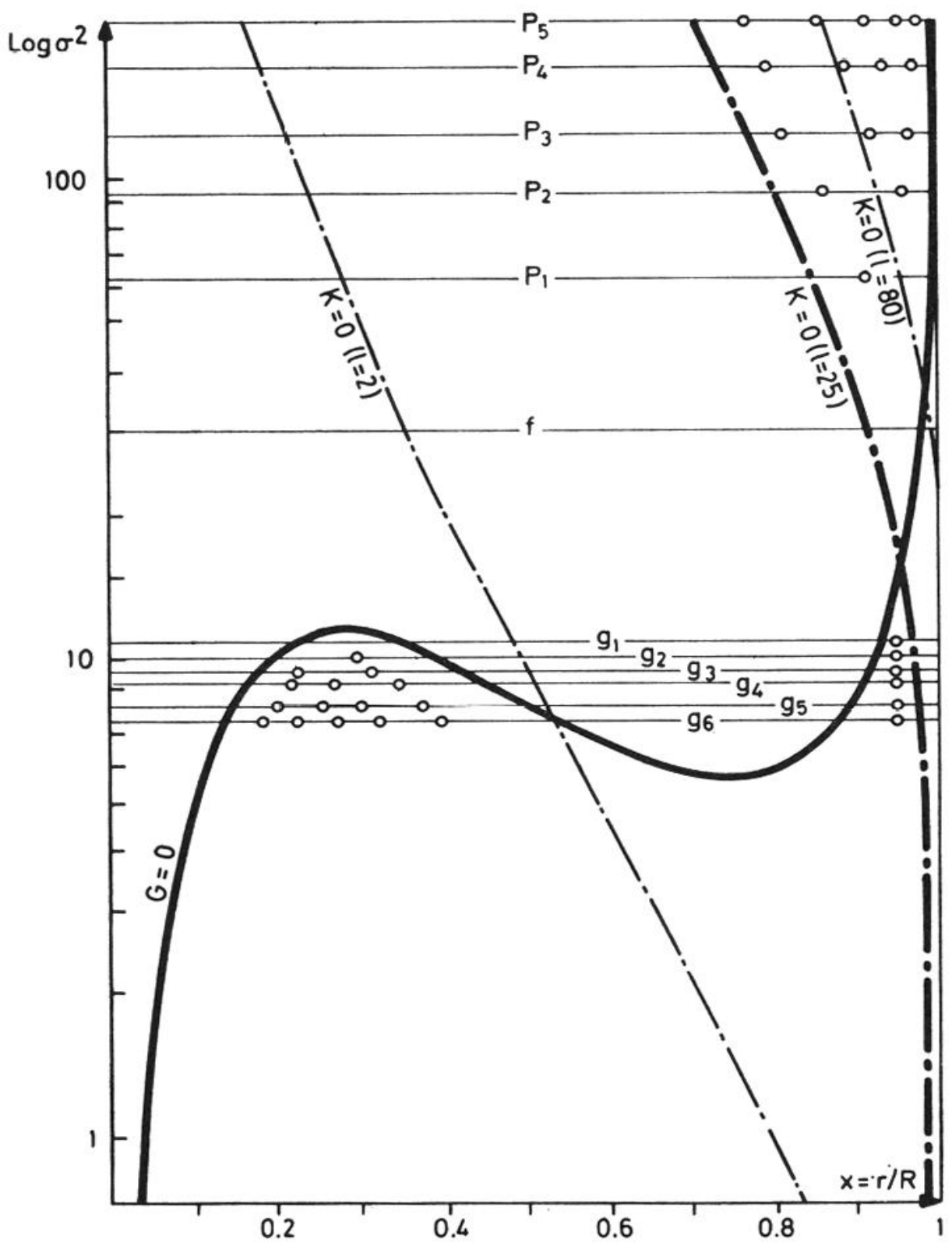

Fig. 1. - Polytrope $n=3$. Courbes $\mathrm{G}=0$ et $\mathrm{K}=0$ pour $l=2, l=25$ et $l=80$. Les positions des nœuds de $\delta r$ des modes $g, p$ pour $l=25$ sont indiqués par de petits cercles. 
D'autre part, les représentations asymptotiques (voir par exemple Tassoul and Tassoul 1968) pour $\sigma^{2}$ très grand (modes $p$ ) ou $\sigma^{2}$ très petit (modes $g$ ) ne peuvent donner qu'une idée fort sommaire du comportement des premiers modes pour $l$ très grand. En effet, bornonsnous à un modèle simple où $\mathrm{A} \leqslant 0$ dans tout l'intervalle $0 \leqslant r \leqslant \mathrm{R}$. On sait que la représentation asymptotique des modes $p$ est valable si $\sigma_{p}^{2}>4 \pi \mathrm{G} \rho_{c} l / 3$. Mais pour les premiers modes $p, \sigma_{p}^{2}$ varie aussi comme $l$ (voir plus loin) et le domaine de validité des formules asymptotiques (c'est-à-dire la fraction des modes à laquelle elles s'appliquent) reste donc relativement constante quand $l$ augmente.

Par ailleurs, à partir du centre la fonction propre asymptotique des modes $p$ pour $(\delta \rho / \rho)$ se comporte comme $\mathrm{J}_{l+1 / 2}\left(\tau_{p}\right)$ où

$$
\tau_{p}=\int_{0}^{r}(d r / \sqrt{\gamma p / \rho)} .
$$

On sait que le premier zéro de $\mathrm{J}_{l+1 / 2} \mathrm{~s}$ 'écarte rapidement de l'origine quand $l$ augmente et comme l'argument $\tau_{p}$ ne grandit que comme $l^{1 / 2}$ on s'attend à ce que les nœuds des modes $p$ s'écartent aussi du centre quand $l$ augmente. Ceci se dégage également de l'équation pour $y=r \rho^{-1 / 2} p$, que l'on peut obtenir par élimination de $v$ entre (1) et (2) et qui pour $\sigma^{2}$ très grand (ceci permet de négliger $\sigma^{2}$ devant $\mathrm{Ag}$ sauf près de la surface) s'écrit:

$$
\frac{d^{2} y}{d r^{2}}-\left(\frac{l(l+1)}{r^{2}}-\frac{\sigma^{2} \rho}{\gamma p}+\frac{1 d^{2} \chi}{\chi d r^{2}}\right) y=0 ; \chi=r p^{1 / \gamma} / \sqrt{\rho} .
$$

On voit que le signe du coefficient de $y$ sera positif dans une région centrale d'autant plus étendue que $l$ est plus grand (même si $\sigma_{p}^{2}$ varie comme $l$ ) ce qui exclut l'existence de zéro de $y$ dans cette région.

Pour les modes $g$, le comportement de $\delta \rho / \rho$ aux environs du centre est encore en $\mathbf{J}_{l+1 / 2}\left(\tau_{g}\right)$, mais ici l'argument $\tau_{g}$ qui est proportionnel à $\sqrt{l(l+1)} / \sigma_{g}$ tend à grandir aussi comme $l$ puisque $\sigma_{g}$ ne grandit que très lentement avec $l$ (voir plus loin ou formule asymptotique pour $\sigma_{g}$ ). Dans le cas des modes $g$, il n'y a donc aucune indication que les nœuds aient une tendance à s'écarter du centre quand $l$ augmente.

Ainsi une discussion assez complète, particulièrement des premiers modes non radiaux de degré $l$ élevé semble utile. Nous la présentons ici pour le modèle standard (polytrope $n=3$ ) qui constitue encore, 
du point de vue qui nous intéresse ici, un modèle assez caractéristique de la constitution des étoiles du moins sur la séquence principale. Une discussion semblable pour des modèles à très grande concentration massique tels que ceux que l'on rencontre au cours de l'évolution stellaire serait aussi fort désirable étant donné les particularités que présentent dans ce cas les fonctions propres des premiers modes $p, g$ et $f$.

\section{RECHERCHE DES MODES NON RADIAUX DU MODÈLE STANDARD}

Nous avons recherché les premiers modes $f, p$ et $g$ du polytrope $n=3$ pour des valeurs du degré $l$ des harmoniques sphériques $\mathrm{Y}_{l}^{m}$ variant de $l=2$ à 200 pour les modes $f$ et $p$ et de 2 à 50 pour les modes $g$. Vu les valeurs élevées de $l$ considérées ici, nous avons négligé la perturbation du potentiel gravifique et intégré le système du second ordre suivant:

$$
\begin{gathered}
\frac{d z_{1}}{d r}=-\frac{l+1}{r}\left(z_{1}-\frac{l}{\sigma^{2}} z_{2}\right)-\frac{1 d p}{\gamma p d r} z_{1}-\frac{\rho r}{\gamma p} z_{2} \\
\frac{d z_{2}}{d r}=\frac{\sigma^{2}}{r}\left(z_{1}-\frac{l}{\sigma^{2}} z_{2}\right)+\mathrm{A} g \cdot \frac{z_{1}}{r}-\mathrm{A} z_{2}
\end{gathered}
$$

$z_{1}$ et $z_{2}$ étant liés à $\delta r$ et $p^{\prime}$ par

$$
\delta r=r^{l-1} z_{1} ; p^{\prime}=\rho r^{l} z_{2} .
$$

Le calcul des valeurs propres pour $l=10$ (voir tableaux I et IV) à partir du système complet du quatrième ordre (Robe 1968) montre d'ailleurs qu'elles coïncident presque avec celles déduites du système (5-6). Pour l'intégration numérique, les variables $z_{i}$, qui se réduisent à des constantes au centre, sont préférables aux variables $v$ et $w$ cidessus qui se comportent respectivement comme $r^{l+1}$ et $r^{l}$ au voisinage du centre et conduisent rapidement lorsque $l$ croît à des instabilités numériques. En fait, ce type de difficultés se manifeste encore avec les nouvelles variables lorsque $l \geqslant 300$; elles proviennent alors des coefficients des singularités $(r=0)$ des équations (5-6); ces coefficients étant respectivement proportionnels à $(l+1)$ et $\sigma^{2}$ (donc aussi à $l$ pour les modes $p$ comme on le verra) et de signes opposés; si bien que, lorsque $l$ devient grand, une petite erreur sur $z_{1}$ et $z_{2}$ est immé- 
diatement amplifiée avec des effets systématiquement de signes contraires sur $z_{1}$ et $z_{2}$.

\section{a. MODES $p$ ET $f$}

Le tableau I indique les valeurs propres $\sigma^{2}$ calculées; le tableau II les positions des nœuds du déplacement radial correspondant.

TABleau I. - Carré des pulsations $\sigma$ en fonction de $l$. Modes $f$ et $p\left(\sigma^{2}\right.$ en unités $G M / R^{3}$ ).

\begin{tabular}{c|c|c|c|c|c|c|c}
\hline \hline \multirow{2}{*}{ degré $l$} & \multicolumn{7}{|c}{ modes } \\
\cline { 2 - 7 } & $p_{6}$ & $p_{5}$ & $p_{4}$ & $p_{3}$ & $p_{2}$ & $p_{1}$ & $f$ \\
\hline 2 & 109,9 & 84,98 & 63,29 & 44,72 & 29,36 & 17,37 & 9,480 \\
$2\left(^{*}\right)$ & 104,9 & 80,55 & 59,42 & 41,47 & 26,72 & 15,26 & 8,180 \\
5 & $\ldots$ & 104,8 & 79,50 & 57,47 & 38,67 & 23,12 & 10,84 \\
10 & 168,8 & 134,8 & 104,1 & 76,95 & 52,22 & 32,79 & 15,05 \\
$10\left(^{*}\right)$ & & & 103,6 & 76,73 & 53,10 & 32,74 & \\
25 & & 218,1 & 174,0 & 133,4 & 96,23 & 62,17 & 29,69 \\
50 & 427,5 & 356,9 & 290,0 & 226,8 & 167,6 & 111,2 & 54,59 \\
100 & 737,0 & 624,8 & 516,1 & 410,9 & 308,9 & 209,0 & 104,6 \\
150 & 1049 & 694,9 & 743,0 & 595,3 & 450,5 & 306,0 & 154,5 \\
200 & 1359 & 1163 & 969,8 & 779,3 & 613,2 & 406,1 & 204,5
\end{tabular}

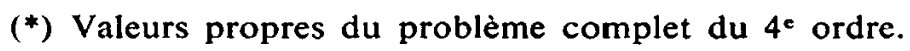

Tableau II. - Positions $x_{i}$ des nœuds de $\delta r$ en fonction de $l$. Modes $p_{1}, p_{2}$ et $p_{5}$.

\begin{tabular}{|c|c|c|c|c|c|c|c|c|}
\hline \multirow{2}{*}{$l$} & \multirow{2}{*}{$\frac{\operatorname{mode} p_{1}}{x_{1}}$} & \multicolumn{2}{|c|}{ mode $p_{2}$} & \multicolumn{5}{|c|}{$\operatorname{mode} p_{5}$} \\
\hline & & $x_{1}$ & $x_{2}$ & $x_{1}$ & $x_{2}$ & $x_{3}$ & $x_{4}$ & $x_{5}$ \\
\hline 2 & 0.675 & 0.54 & 0.83 & 0.36 & 0.61 & 0.77 & 0.875 & 0.948 \\
\hline 10 & 0.84 & 0.763 & 0.913 & 0.62 & 0.764 & 0.857 & 0.922 & 0.967 \\
\hline 50 & 0.958 & 0.924 & 0.9728 & 0.851 & 0.910 & 0.946 & 0.9709 & 0.9879 \\
\hline 100 & 0.9752 & 0.96 & 0.9852 & 0.916 & 0.945 & 0.965 & 0.9834 & 0.993 \\
\hline 150 & 0.9832 & 0.9714 & 0.9899 & 0.94 & 0.966 & 0.9784 & 0.9884 & 0.9962 \\
\hline 200 & 0.9873 & 0.9797 & 0.9926 & 0.957 & 0.9722 & 0.9834 & 0.9911 & 0.9963 \\
\hline
\end{tabular}

A partir de $l=50$, les valeurs propres $\sigma^{2}$ deviennent à peu près proportionnelles à $l$ et les nœuds se rapprochent de plus en plus de la 
surface. Il en est rigoureusement ainsi dans le cas du modèle homogène (Pekeris 1938, Sauvenier-Goffin 1951). Les résultats ci-dessus suggèrent qu'il doit en être approximativement ainsi du moins pour tout modèle dont la concentration massique n'est pas trop élevée.

Par ailleurs, d'après le tableau III qui reflète bien l'allure des divers modes calculés, les diverses perturbations ne sont relativement importantes que dans le voisinage immédiat de la surface. Ce comportement est aussi observé pour le modèle homogène (Boury 1974, Rossius 1974). Ainsi, les modes $p$ et $f$, pour $l$ grand, sont limités aux couches superficielles et on peut considérer celles-ci comme planes, l'approximation étant d'autant plus justifiée que $l$ est grand.

Tableau III. - Modes $p$. Amplitude du déplacement radial $\delta r$ $(x=r / \mathrm{R} ; \delta r=1$ en $x=1)$.

\begin{tabular}{|c|c|c|c|c|c|c|c|}
\hline \multicolumn{2}{|c|}{$\operatorname{mode} p_{3} ; l=2$} & \multicolumn{2}{|c|}{ mode $p_{4} ; l=25$} & \multicolumn{2}{|c|}{$\operatorname{mode} p_{3} ; l=200$} & \multicolumn{2}{|c|}{$\operatorname{mode} p_{6} ; l=200$} \\
\hline$x$ & $\delta r$ & $x$ & $\delta r$ & $x$ & $\delta r$ & $x$ & $\delta r$ \\
\hline 0. & 0. & 0. & 0. & 0. & 0. & 0. & 0. \\
\hline 0,1 & $-0.18 \mathrm{E}-3$ & 0,1 & $-0,13 E-23$ & 0,1 & $-0,84 \mathrm{E}-196$ & 0,1 & $+0,50 \mathrm{E}-193$ \\
\hline 0,2 & $-0,38 \mathrm{E}-3$ & 0,2 & $-0,18 \mathrm{E}-16$ & 0,35 & $-0,30 \mathrm{E}-76$ & 0,4 & $+0,33 \mathrm{E}-73$ \\
\hline 0,3 & $-0,57 \mathrm{E}-3$ & 0,3 & $-0,28 \mathrm{E}-12$ & 0,50 & $-0,64 \mathrm{E}-57$ & 0,6 & $+0,35 \mathrm{E}-38$ \\
\hline 0,33 & $-0,60 \mathrm{E}-3$ & 0,4 & $-0,29 \mathrm{E}-9$ & 0,70 & $-0,80 \mathrm{E}-28$ & 0,8 & $+0,48 \mathrm{E}-14$ \\
\hline 0,34 & $-0,61 \mathrm{E}-3$ & 0,5 & $-0,69 \mathrm{E}-7$ & 0,90 & $-0,67 \mathrm{E}--7$ & 0,94 & $+0,88 \mathrm{E}-4$ \\
\hline 0,35 & $-0,60 \mathrm{E}-3$ & 0,6 & $-0,54 \mathrm{E}-5$ & 0,95 & $-0,28 \mathrm{E}-3$ & 0,95 & $-0,16 \mathrm{E}-3$ \\
\hline 0,4 & $-0,50 \mathrm{E}-3$ & 0,7 & $-0,15 \mathrm{E}-3$ & 0,96 & $-0,70 \mathrm{E}-3$ & 0,97 & $+0,84 \mathrm{E}-3$ \\
\hline 0,45 & $-0,14 \mathrm{E}-3$ & 0,78 & $-0,75 \mathrm{E}-3$ & 0,97 & $+0,74 \mathrm{E}-4$ & 0,9724 & $+0,11 \mathrm{E}-2$ \\
\hline 0,5 & $+0,59 \mathrm{E}-3$ & 0,79 & $-0,79 \mathrm{E}-3$ & 0,98 & $+0,51 \mathrm{E}-2$ & 0,9728 & $+0,11 \mathrm{E}-2$ \\
\hline 0,6 & $+0,33 \mathrm{E}-2$ & 0,80 & $-0,76 \mathrm{E}-3$ & 0,981 & $+0,53 \mathrm{E}-2$ & 0,974 & $+0,11 \mathrm{E}-2$ \\
\hline 0,66 & $+0,46 \mathrm{E}-2$ & 0,85 & $+0,15 \mathrm{E}-2$ & 0,982 & $+0,52 \mathrm{E}-2$ & 0,976 & $+0,64 \mathrm{E}-3$ \\
\hline 0,67 & $+0,47 \mathrm{E}-2$ & 0,88 & $+0,48 \mathrm{E}-2$ & 0,984 & $+0,35 \mathrm{E}-2$ & 0,9772 & $+0,14 \mathrm{E}-3$ \\
\hline 0,68 & $+0,46 \mathrm{E}-2$ & 0,89 & $+0,54 \mathrm{E}-2$ & 0,99 & $-0,19 \mathrm{E}-1$ & 0,9776 & $-0,65 \mathrm{E}-4$ \\
\hline 0,7 & $0,40 \mathrm{E}-2$ & 0,90 & $+0,50 \mathrm{E}-2$ & 0,991 & $-0,23 \mathrm{E}-1$ & 0,98 & $-0,15 \mathrm{E}-2$ \\
\hline 0,80 & $-0,12 \mathrm{E}-1$ & 0,94 & $-0,18 \mathrm{E}-1$ & 0,992 & $-0,25 \mathrm{E}-1$ & 0,9824 & $-0,26 \mathrm{E}-2$ \\
\hline 0,85 & $-0,24 \mathrm{E}-1$ & 0,948 & $-0,24 \mathrm{E}-1$ & 0,9924 & $-0,24 \mathrm{E}-1$ & 0,9828 & $-0,26 \mathrm{E}-2$ \\
\hline 0,86 & $-0,23 \mathrm{E}-1$ & 0,95 & $-0,25 \mathrm{E}-1$ & 0,994 & $-0,78 \mathrm{E}-1$ & 0,9861 & 0. \\
\hline 0,90 & $+0,35 \mathrm{E}-2$ & 0,952 & $-0,25 \mathrm{E}-1$ & 0,996 & $+0,79 \mathrm{E}-1$ & 0,99 & $+0,72 \mathrm{E}-2$ \\
\hline 0,92 & $+0,50 \mathrm{E}-1$ & 0,97 & $+0,18 \mathrm{E}-1$ & 0,998 & $+0,34$ & 0,9904 & $+0,72 \mathrm{E}-2$ \\
\hline 0,95 & $+0,20$ & 0,98 & $+0,13$ & 0,9992 & $+0,67$ & 0,9925 & 0. \\
\hline 0,97 & $+0,41$ & 0,99 & $+0,41$ & 0,9996 & $+0,81$ & 0,9952 & $-0,27 \mathrm{E}-1$ \\
\hline 0,99 & $+0,76$ & 0,996 & $+0,71$ & & & 0,9956 & $-0,2 \mathrm{E}-18$ \\
\hline 1 . & 1. & 1. & 1. & 1. & 1. & $\begin{array}{c}0,9968 \\
1 .\end{array}$ & 1. \\
\hline
\end{tabular}




\section{b. MODEs $g$}

Au tableau IV sont indiquées les valeurs propres calculées; au tableau $V$ la position des nœuds de quelques modes et au tableau VI les amplitudes du déplacement radial de modes représentant bien l'ensemble des modes calculés.

TABleau IV. - Carré des Pulsations, $\sigma^{2}$, des modes $g$. $\left(\sigma^{2}\right.$ en unités $\left.\mathrm{GM} / \mathrm{R}^{3}\right)$.

\begin{tabular}{c|c|c|c|c|c|c|c}
\hline \hline \multirow{2}{*}{ degré $l$} & \multicolumn{7}{|c}{ modes $g$} \\
\cline { 2 - 8 } & $g_{10}$ & $g_{6}$ & $g_{5}$ & $g_{4}$ & $g_{3}$ & $g_{2}$ & $g_{1}$ \\
\hline 2 & 0,3230 & 0,7243 & 0,9458 & 1,289 & 1,864 & 2,939 & 5,326 \\
$2\left(^{*}\right)$ & & 0,7188 & 0,9360 & 1,271 & 1,823 & 2,828 & 4,915 \\
3 & & 1,253 & 1,600 & 2,117 & 2,935 & 4,346 & 7,070 \\
4 & & 1,813 & 2,268 & 2,920 & 3,902 & 5,482 & 8,228 \\
5 & & 2,369 & 2,908 & 3,654 & 4,729 & 6,356 & 8,978 \\
10 & 2,901 & 4,630 & 5,296 & 6,118 & 7,155 & 8,490 & 10,26 \\
$10\left(^{*}\right)$ & & & & & 7,124 & 8,445 & 10,19 \\
15 & 4,256 & 5,970 & 6,600 & 7,371 & 8,265 & 9,338 & 10,65 \\
25 & 5,883 & 7,500 & 8,070 & 8,670 & 9,345 & 10,10 & 10,97 \\
50 & & & & 8,900 & 10,31 & 10,74 & 11,21
\end{tabular}

(*) Valeurs propres du problème complet du $4^{e}$ ordre.

Tableau V. - Positions $x_{i}$ des nœuds de $\delta r$ en fonction de $l$. Modes $g_{1}, g_{2}, g_{4}$.

\begin{tabular}{r|c|c|c|c|c|c|c}
\hline \hline \multirow{2}{*}{$l$} & \multirow{2}{*}{ mode $g_{1}$} & \multicolumn{2}{|c|}{ mode $g_{2}$} & \multicolumn{5}{|c}{ mode $g_{4}$} \\
\cline { 2 - 7 } \cline { 5 - 7 } & $x_{1}$ & $x_{1}$ & $x_{2}$ & $x_{1}$ & $x_{2}$ & $x_{3}$ & $x_{4}$ \\
\hline 2 & 0,41 & 0,21 & 0,53 & 0,125 & 0,21 & 0,35 & 0,67 \\
10 & 0.78 & 0,28 & 0,81 & 0,20 & 0,275 & 0,38 & 0,855 \\
25 & 0,95 & 0,28 & 0,95 & 0,226 & 0,28 & 0,351 & 0,957
\end{tabular}

Souffrin (1972) a démontré, qu'en module, les valeurs propres de tous les modes non radiaux sont des fonctions croissantes de $l$ et de plus, que $\left|\sigma^{2}\right| \leqslant \operatorname{Max}|\mathrm{A} g|$ dans le cas des modes $g^{-}$associés à une zone convectivement instable $(\mathrm{A}>0)$.

Dans le modèle considéré ici, $A$ est partout négatif et tend vers $-\infty$ à la surface. Cependant, les valeurs $\sigma_{g}^{2}$ trouvées sont toutes inférieures 
Tableau VI. - Modes $g$. Amplitude du déplacement radial $\delta r$. $(x=r / \mathrm{R} ; \delta r=1$ en $x=1)$.

\begin{tabular}{|c|c|c|c|c|c|c|c|}
\hline \multicolumn{4}{|c|}{ mode $g_{1}$} & \multicolumn{3}{|c|}{ mode $g_{3}$} & \multirow{2}{*}{$\begin{array}{c}\text { mode } g_{6} \\
\delta r(l=25)\end{array}$} \\
\hline$x$ & $\delta r(l=2)$ & $\delta r(l=10)$ & $\delta r(l=25)$ & $\delta r(l=2)$ & $\delta r(l=10)$ & $\delta r(l=25)$ & \\
\hline 0 & 0. & 0. & 0. & 0. & 0. & 0. & 0. \\
\hline 0,05 & $-0,17$ & $-0,35 \mathrm{E}-3$ & $-0,35 \mathrm{E}-6$ & $-0,15 \mathrm{E}+1$ & $-0,17 \mathrm{E}-2$ & $-0,48 \mathrm{E}-6$ & $+0.55 \mathrm{E}-6$ \\
\hline 0,1 & $-0,29$ & $-0,84 \mathrm{E}-3$ & $-0,82$ & $-0,12 \mathrm{E}+1$ & $-0,27$ & $-0,78$ & $+0,47$ \\
\hline 0,13 & & $-0,46$ & $-0,83 \mathrm{E}+2$ & $-0,57$ & $-0,10 E+1$ & $-0,54 \mathrm{E}+2$ & $+0,16 \mathrm{E}+2$ \\
\hline 0,15 & $-0,31$ & $-0,10 \mathrm{E}+1$ & $-0,70 E+3$ & $-0,14$ & $-0,16 \mathrm{E}+1$ & $-0,54 \mathrm{E}+2$ & $+0,16 \mathrm{E}+2$ \\
\hline 0,17 & & & & $+0,18$ & $-0,18 \mathrm{E}+1$ & & \\
\hline 0,20 & $-0,28$ & $-0,32 \mathrm{E}+1$ & $-0,17 \mathrm{E}+5$ & $+0,41$ & $-0,11 \mathrm{E}+1$ & $-0,24 \mathrm{E}+4$ & $-0,26 \mathrm{E}+2$ \\
\hline 0,21 & $-0,26$ & $-0,36 \mathrm{E}+1$ & $-0,25 E+5$ & $+0,43$ & $-0,74$ & $-0,25 E+4$ & $-0,46 E+2$ \\
\hline 0,24 & $-0,22$ & $-0,46 \mathrm{E}+1$ & $-0,51 \mathrm{E}+5$ & $+0,34$ & $+0,50$ & $-0,52 \mathrm{E}+3$ & $+0,15 \mathrm{E}+2$ \\
\hline 0,25 & $-0,21$ & $-0,48 \mathrm{E}+1$ & $-0,59 E+5$ & $+0,28$ & $+0,80$ & $+0,42 \mathrm{E}+3$ & $+0,37 \mathrm{E}+2$ \\
\hline 0,26 & $-0,19$ & $-0,49 \mathrm{E}+1$ & $-0,64 \mathrm{E}+5$ & $+0,22$ & $+0,10 \mathrm{E}+1$ & $+0,12 \mathrm{E}+4$ & $+0,39 \mathrm{E}+2$ \\
\hline 0,28 & $-0,16$ & $-0,49 E+1$ & $-0,68 E+5$ & $+0,78 \mathrm{E}-1$ & $+0,11 \mathrm{E}+1$ & $+0,19 \mathrm{E}+4$ & $-0,12 E+1$ \\
\hline 0,30 & $-0,14$ & $-0,47 \mathrm{E}+1$ & $-0,63 \mathrm{E}+5$ & $-0,58 \mathrm{E}-1$ & $+0,88$ & $+0,12 \mathrm{E}+4$ & $-0.38 E+2$ \\
\hline 0,35 & $-0,70 \mathrm{E}--1$ & $-0,36 \mathrm{E}+1$ & $-0,34 E+5$ & $-0,31$ & $-0,34$ & $-0,17 \mathrm{E}+4$ & $+0,32 \mathrm{E}+2$ \\
\hline 0,37 & $-0,46 \mathrm{E}-1$ & $-0,31 \mathrm{E}+1$ & $-0,24 E+5$ & $-0,36$ & $-0,78$ & $-0,22 \mathrm{E}+4$ & $+0,42 \mathrm{E}+2$ \\
\hline 0,40 & $-0,12 \mathrm{E}-1$ & $-0,24 \mathrm{E}+1$ & $-0,12 \mathrm{E}+5$ & $-0,40$ & $-0,12 \mathrm{E}+1$ & $-0,21 \mathrm{E}+4$ & $+0,76 \mathrm{E}+1$ \\
\hline 0,45 & $+0,38 \mathrm{E}-1$ & $-0,15 \mathrm{E}+1$ & $-0,39 E+4$ & $-0,38$ & $-0,15 \mathrm{E}+1$ & $-0,11 \mathrm{E}+4$ & $-0,56 E+2$ \\
\hline 0,47 & $+0,57 \mathrm{E}-1$ & $-0,12 \mathrm{E}+1$ & $-0,24 \mathrm{E}+4$ & $-0,35$ & $-0,14 \mathrm{E}+1$ & $-0,79 E+3$ & $-0,63 E+2$ \\
\hline 0,50 & $+0,85 \mathrm{E}-1$ & $-0,96$ & $-0,11 \mathrm{E}+4$ & $-0,29$ & $-0,13 E+1$ & $-0,45 E+3$ & $-0,58 \mathrm{E}+2$ \\
\hline 0,55 & $+0,13$ & $-0,60$ & $-0,31 E+3$ & $-0,17$ & $-0,11 \mathrm{E}+1$ & $-0,16 \mathrm{E}+3$ & $-0,35 E+2$ \\
\hline 0,60 & $+0,18$ & $-0,37$ & $-0,89 E+2$ & $-0,36 \mathrm{E}-1$ & $-0,80$ & $-0,55 \mathrm{E}+2$ & $-0,18 E+2$ \\
\hline 0,65 & $+0,23$ & $-0,23$ & $-0,27 \mathrm{E}+2$ & $+0,97 \mathrm{E}-1$ & $-0,58$ & $-0,20 \mathrm{E}+2$ & $-0,85 \mathrm{E}+1$ \\
\hline 0,70 & $+0,29$ & $-0,14$ & $-0,93 E+1$ & $+0,22$ & $-0,40$ & $-0,76 \mathrm{E}+1$ & $-0,41 \mathrm{E}+1$ \\
\hline 0,75 & $+0,36$ & $-0,55 \mathrm{E}-1$ & $-0,35 E+1$ & $+0,35$ & $-0,26$ & $-0,32 \mathrm{E}+1$ & $-0,19 E+1$ \\
\hline 0,80 & $+0,44$ & $+0,30 \mathrm{E}-1$ & $-0,15 E+1$ & $+0,48$ & $-0,12$ & $-0,15 \mathrm{E}+1$ & $-0,13 E+1$ \\
\hline 0,85 & +054 & $+0,14$ & $-0,76 \mathrm{E}+1$ & $+0,60$ & $+0,44 \mathrm{E}-1$ & $-0,85$ & $-0,84 \mathrm{E}+1$ \\
\hline 0,90 & $+0,67$ & $+0,30$ & $-0,37$ & $+0,73$ & $+0,25$ & $-0,48$ & $-0,58$ \\
\hline 0,95 & $+0,82$ & $+0,57$ & $+0,47 \mathrm{E}-1$ & $+0,86$ & $+0,55$ & $-0,45 \mathrm{E}-1$ & $-0,17$ \\
\hline 0,99 & $+0,96$ & $+0,90$ & $+0,72$ & $+0,97$ & $+0,90$ & $+0,70$ & $+0,65$ \\
\hline 1 & 1 & 1 & 1 & 1 & 1 & 1 & 1 \\
\hline
\end{tabular}

au maximum relatif de $|\mathrm{Ag}|$ atteint en $x \simeq 0,28$ et suggèrent que ce maximum pourrait être ici aussi une limite des $\sigma_{g}^{2}$ lorsque $l$ croît. Ceci expliquerait d'ailleurs les difficultés numériques rencontrées dans la recherche de modes $g$ pour $l>50$, car à des valeurs propres $\sigma^{2}$ voisines, correspondraient alors des fonctions propres nettement différentes (par exemple par le nombre de nœuds). En fait, même pour des valeurs de l'ordre de 15 , il faut déjà rechercher les $\sigma^{2}$ avec une précision relative très grande afin de calculer sans erreur les fonctions propres. 
Pour les modes $g$, contrairement au cas des modes $p$ et $f$, les amplitudes des diverses perturbations restent importantes dans tout le modèle. Un net accroissement avec $l$ des amplitudes atteintes à l'intérieur a été observé sur les premiers modes $g\left(g_{1}\right.$ à $\left.g_{5}\right)$; il est le plus marqué sur le mode $g_{1}$ et décroît avec l'ordre du mode ainsi que l'indique le tableau VII. Par contre, du moins pour des valeurs de $l$ considérées ici, si l'amplitude des modes $g_{8}$ à $g_{14}$ reste encore importante dans tout le modèle, on observe pour les modes $g_{10}$ à $g_{14}$ que l'amplitude maximum décroît avec $l$. L'ensemble des résultats du tableau VII suggère cependant que pour un mode $g$ donné, l'amplitude maximum atteinte passe par un minimum puis augmente avec $l$. Ceci est évident pour les modes $g_{1}$ à $g_{8}$.

Tableau VII. - Modes $g$. Maxima de $|\delta r|$ en fonction de $l(\delta r=1$ en $x=1$ ).

\begin{tabular}{|c|c|c|c|c|c|c|c|c|c|c|}
\hline \multirow{2}{*}{$\frac{\text { Modes }}{g_{1}}$} & \multicolumn{2}{|c|}{$l=2$} & \multicolumn{2}{|c|}{$l=4$} & \multicolumn{2}{|c|}{$l=10$} & \multicolumn{2}{|c|}{$l=15$} & \multicolumn{2}{|c|}{$l=25$} \\
\hline & 0.32 & $(0.14)(*)$ & 0.15 & $(0.22)$ & $0.49 E+1$ & $(0.27)$ & $0.14 \mathrm{E}+3$ & $(0.28)$ & $0.68 \mathrm{E}+5$ & $(0.28)$ \\
\hline \multirow[t]{2}{*}{$g_{2}$} & 0.97 & $(0.09)$ & 0.46 & $(0.14)$ & $0.27 \mathrm{E}+1$ & $(0.20)$ & $0.40 \mathrm{E}+2$ & $(0.21)$ & $0.11 \mathrm{E}+5$ & $(0.23)$ \\
\hline & 0.35 & $(0.32)$ & 0.29 & $(0.37)$ & $0.23 \mathrm{E}+1$ & $(0.38)$ & $0.35 \mathrm{E}+1$ & $(0.36)$ & $0.99 E+4$ & $(0.34)$ \\
\hline \multirow[t]{2}{*}{$g_{3}(* *)$} & $0.16 \mathrm{E}+1$ & (0.07) & 0.75 & $(0.11)$ & $0.18 \mathrm{E}+1$ & $(0.17)$ & $0.16 \mathrm{E}+2$ & $(0.19)$ & $0.25 \mathrm{E}+4$ & $(0.21)$ \\
\hline & 0.41 & $(0.42)$ & 0.38 & $(0.45)$ & $0.15 \mathrm{E}+1$ & $(0.45)$ & $0.13 \mathrm{E}+2$ & $(0.41)$ & & \\
\hline \multirow[t]{2}{*}{$g_{4}$} & & & 0.97 & $(0.09)$ & $0.13 \mathrm{E}+1$ & $(0.15)$ & $0.11 \mathrm{E}+2$ & $(0.17)$ & $0.71 \mathrm{E}+3$ & $(0.19)$ \\
\hline & & & 0.43 & $(0.52)$ & $0.11 \mathrm{E}+1$ & $(0.51)$ & $0.91 \mathrm{E}+1$ & $(0.46)$ & $0.61 \mathrm{E}+3$ & $(0.41)$ \\
\hline \multirow[t]{2}{*}{$g_{6}$} & $0.31 \mathrm{E}+1$ & $(0.04)$ & $0.13 \mathrm{E}+1$ & $(0.07)$ & 0.76 & $(0.12)$ & $0.18 \mathrm{E}+1$ & $(0.14)$ & $0.70 \mathrm{E}+2$ & $(0.17)$ \\
\hline & 0.47 & $(0.58)$ & 0.48 & $(0.61)$ & 0.70 & $(0.64)$ & $0.17 \mathrm{E}+1$ & $(0.56)$ & $0.63 E+2$ & $(0.47)$ \\
\hline \multirow[t]{2}{*}{$g_{8}$} & & & & & 0.57 & $(0.10)$ & 0.63 & (0.13) & $0.90 \mathrm{E}+1$ & $(0.15)$ \\
\hline & & & & & 0.60 & $(0.73)$ & 0.78 & $(0.70)$ & $0.83 E+1$ & $(0.52)$ \\
\hline \multirow[t]{2}{*}{$g_{10}$} & $0.42 \mathrm{E}+1$ & $(0.025)$ & & & 0.49 & $(0.09)$ & 0.34 & $(0.11)$ & $0.11 \mathrm{E}+1$ & $(0.14)$ \\
\hline & 0.78 & $(0,076)$ & & & 0.55 & $(0.78)$ & 0.61 & $(0.80)$ & $0.12 E+1$ & (0.61) \\
\hline \multirow[t]{2}{*}{$g_{14}$} & & & & & 0.44 & $(0.07)$ & & & $0.88 \mathrm{E}-1$ & $(0.12)$ \\
\hline & & & & & 0.52 & $(0.85)$ & & & 0.55 & $(0.92)$ \\
\hline$g_{17}$ & $\begin{array}{l}0.45 \mathrm{E}+1 \\
0.86\end{array}$ & $\begin{array}{l}(0.016) \\
(0.046)\end{array}$ & & & & & & & & \\
\hline
\end{tabular}

(*) Entre parenthèses, abscisse $x$ de l'extremum.

(**) A partir de $g_{3}$. seuls les deux maxima les plus élevés sont indiqués.

Enfin, en accord avec les conjectures évoquées dans l'introduction, on constate, pour $l=25$ notamment, que si le mode $g_{1}$ a son unique nœud près de la surface, les autres modes ont toujours un nœud près de la surface et les autres nœuds dans la région centrale permise (voir aussi figure 1). 


\section{Discussion et CONCLUSIONS}

Pour $l$ élevé, le mode $f$ et les premiers modes $p$ sont limités aux couches extérieures du modèle et on peut traiter ces dernières comme planes. Les variations des amplitudes calculées ici suggèrent d'adopter alors les conditions aux limites $\delta r=0, \delta p=0$ à la base de la couche. $\mathrm{Vu}$ la distribution des nœuds et des amplitudes, l'approximation plane n'est absolument pas valable pour les modes $g$ du modèle considéré ici. Il est vrai que l'allure de ces modes dépend essentiellement du facteur $\mathrm{Ag}$ et que dans d'autres modèles, l'approximation pourrait être justifiée; par exemple pour les modes $g^{+}$ou $g^{-}$associés respectivement à une zone radiative située dans les couches extérieures et bornées au moins vers le bas par une couche convective ou à une zone convective située entre deux zones radiatives.

Les valeurs propres de tous les modes radiaux croissent avec $l$. Comme celles des modes $p$ deviennent alors à peu près porportionnelles à $l$, le facteur $l(l+1) / \sigma^{2}$ dans l'équation (1), ne devient négligeable devant $\rho r^{2} / \gamma p$ que pour des modes d'ordre de plus en plus élevé et comme nous l'avons déjà signalé, ceci implique que les formules asymptotiques usuelles ( $\sigma_{p}^{2}$ très grand) ne seront valables que pour des modes d'ordre de plus en plus élevé quand $l$ augmente.

Le Tableau VIII donne les périodes des divers modes dans le cas du soleil $\left(\bar{\rho}=1,4109 \mathrm{gr} / \mathrm{cm}^{3}\right)$. Si l'on admet que les zones associées aux

Tableau Vili. - Période T des modes $g, f$ et $p$ en fonction de $l$. ( $T$ en secondes; $\bar{\rho}=1,4109 \mathrm{gr} / \mathrm{cm}^{3}$ ).

\begin{tabular}{c|c|c|c|c|c|c|c|c}
\hline \hline$l$ & $g_{2}$ & $g_{1}$ & $f$ & $p_{1}$ & $p_{2}$ & $p_{3}$ & $p_{4}$ & $p_{5}$ \\
\hline 2 & 5837 & 4336 & 3250 & 2401 & 1846 & 1497 & 1252 & 1086 \\
10 & 3434 & 3124 & 2519 & 1741 & 1372 & 1141 & 980,8 & 862 \\
25 & & & 1836 & 1269 & 1020 & 866,4 & 758,6 & 677,6 \\
50 & 3053 & 2988 & 1354 & 949 & 773 & 664,5 & 587,6 & 529,6 \\
100 & & & 978,6 & 692,2 & 569,3 & 493,6 & 440,5 & 400,3 \\
200 & & & 699,7 & 496,5 & 404,1 & 358,5 & 321,3 & 293,3 \\
$400\left({ }^{1}\right)$ & & & 495 & 351 & 286 & 253 & 227 & \\
$800\left({ }^{(}\right)$ & & & 349 & 248 & 202 & 179 & 160 & \\
$400\left({ }^{2}\right)$ & & & 502 & 263 & 191 & & & \\
$800\left(^{(2)}\right.$ & & & 354 & 186 & 134 & & &
\end{tabular}

( $\left.{ }^{1}\right)$ Valeurs extrapolées.

$\left({ }^{2}\right)$ Cas du modèle homogène compressible, valeurs exactes. 
mouvements observés par Leighton ont des dimensions linéaires de l'ordre de 5000 à $10.000 \mathrm{~km}$, les oscillations non radiales $\mathrm{Y}_{l}^{m}$ qui pourraient y correspondre seraient caractérisées par $m$ variant de 400 à 200 et $l$ de 800 à 400 [Y $Y_{l}^{m}$ a $2 m$ nœuds suivant un parallèle et $2(l-m)$ nœuds suivant un méridien]. Pour ces valeurs de $l$, les périodes des premiers modes $p$ trouvées en extrapolant les résultats obtenus pour $l=200$, ainsi que les valeurs calculées rigoureusement dans le cas $\mathrm{du}$ modèle homogène, ne sont pas incompatibles avec les périodes de l'ordre de 5 minutes observées par Leighton et al. Il serait intéressant d'étudier, comme Wolff l'a déjà fait pour $l=40$ et $l=50$, plus systématiquement la stabilité vibrationnelle de ces modes quand $l$ augmente.

Finalement, le net accroissement avec $l$ des amplitudes des premiers modes $g$ et surtout du mode $g_{1}$ suggère que ces modes pourraient être vibrationnellement instables dans certains modèles et nous nous proposons d'étudier ce problème dans une prochaine note.

\section{RÉFÉRENCES}

Boury, A., 1974, Communication privée.

Cowling, T. G., 1941, M.N.R.A.S. 101, 368.

LEDOUX, P., 1974, «Non Radial Oscillations » in Stellar Stability and Evolution

I.A.U. Symposium $n^{\circ}$ 59. Ed. P. Ledoux, A. Noels, A. Rogers.

Ledoux, P. and Walraven, Th., 1958, Handbuch der Physik, 51, 509.

MACKenzie, J. F., 1971, Astr. Astrophys, 15, 450.

Michalitsanos, A. G., 1973, Earth and Extraterr. Sc. 2, 125.

Pekeris, C. L., 1938, Ap. J. 88, 189.

Rossıus, N., 1974, Mémoire de licence, Université de Liège, 1974.

Sauvenier-Goffin, 1951, Bull. Soc. Roy. Sc. Liège, 20, 20.

Scuflaire, R., 1974, Astr. Astrophys. 36, 107.

SMEYers, P., 1970, Astr. Astrophys. 7, 204,

Souffrin, P., Grisvard, P., Zerner, M., 1972, Astr. Astron. 17, 309.

Stein, R. F. and Leibacher, R., 1974, «Waves in the solar Atmosphere », Ann.

Rev. of Astron. and Astrophys. 12, 407.

Tassoul and Tassoul, 1968, Ann. Astro., 31, 251.

Thomas Clark and Clark, 1971, Astrophysical Letters 16, 51.

WolfF, Ch., 1972a, Ap. J. 176, 833; 1792b, Ap. J. 177, L87.

Worral, G., 1972, Ap. J. 172, 749. 